ÉGYPTE monde arabe

\section{Égypte/Monde arabe}

$10 \mid 1992$

Partage de l'eau dans le monde arabe

\title{
Partage de l'eau dans le monde arabe (suite)
}

\section{(2) OpenEdition}

1 Journals

Édition électronique

URL : https://journals.openedition.org/ema/1416

DOI : 10.4000/ema.1416

ISSN : 2090-7273

\section{Éditeur}

CEDEJ - Centre d'études et de documentation économiques juridiques et sociales

\section{Édition imprimée}

Date de publication : 30 juin 1992

Pagination : 79-108

ISSN : 1110-5097

\section{Référence électronique}

«Partage de l'eau dans le monde arabe (suite) », Égypte/Monde arabe [En ligne], 10 | 1992, mis en ligne le 08 juillet 2008, consulté le 07 juillet 2022. URL : http://journals.openedition.org/ema/1416 ; DOI : https://doi.org/10.4000/ema.1416

Ce document a été généré automatiquement le 7 juillet 2022.

Tous droits réservés 


\section{Partage de l'eau dans le monde arabe (suite)}

Pour compléter le dossier ouvert en première partie du présent numéro sur la question du partage de l'eau dans le monde arabe (voir « décrypter » p. 7 à 75), nous présentons dans les pages qui suivent une sélection d'articles ou extraits d'articles traduits, tirés de la presse arabophone et anglophone :

- Partage de l'eau et droit islamique : confrontation entre les principales orientations de la législation internationale contemporaine quant aux règles qui régissent l'usage des grands fleuves, et celles de la jurisprudence islamique.

- Un fleuve pour neuf nations : conséquences éventuelles, pour les pays riverains du Nil et notamment l'Égypte, des projets hydrologiques éthiopiens.

- Israël : des besoins croissants en eau : les diverses stratégies mises en œuvre par l'État hébreu pour faire face à des besoins hydriques rendus cruciaux par le développement de l'agriculture et par l'arrivée de nouveaux immigrants.

- Turquie : une inépuisable réserve d'eau : les projets hydrauliques turcs de grande envergure (développement de l'Anatolie du sud-est, pipe-line de la paix) et leurs effets potentiels sur le partage de l'eau dans la région ; l'exportation des eaux turques vers lés pays arabes voisins est-elle la solution de l'avenir?

- Ressources hydrologiques de la Jordanie : du développement de celles-ci dépend l'évolution des secteurs essentiels de l'économie jordanienne.

\section{Partage de l'eau et droit islamique}

Dr Tal'at al-Ghunaymi

\section{Al-Charq al-Awsat 28 décembre 1989}

4 Depuis fort longtemps, la question du partage des eaux fluviales préoccupe les communautés internationales. Dire que l'édification d'un État va de pair avec son accès à l'eau n'est nullement exagéré. C'est sur les rives des fleuves que sont nées les civilisations, qu'ont été construites les villes et que l'homme a pu goûter la stabilité. L'Histoire nous apprend comment l'homme, pour tirer profit des eaux du fleuve et des 
erres qu'il baigne, a construit des barrages, creusé des canaux et détourné son cours. $[\ldots]$

5 J'exposerai sommairement les positions de la législation internationale contemporaine et celles de la jurisprudence islamique sur cette question. Le problème est de déterminer si, en l'absence d'entente entre les différents États riverains d'un même fleuve, il existe des règlements internationaux régissant l'utilisation des eaux fluviales et définissant les relations entre ces pays. Les spécialistes en droit international contemporain divergent sur les principes déterminant l'usage des fleuves à vocation économique internationale. En voici, en résumé, les principales orientations avec, en regard, la théorie islamique.

\section{La théorie de la souveraineté absolue}

6 Chaque pays, en tant qu'État souverain, possède le droit à l'usage libre et intégral des eaux fluviales. Autrement dit, il les possède dans sa totalité et est libre de les utiliser à son gré et sans aucune limite. Cette théorie est rejetée par les conceptions islamiques qui considèrent que le droit de jouissance absolue d'un État sur des eaux fluviales internationales entraîne l'anarchie. La jurisprudence islamique, elle, puise sa source dans les paroles du Prophète : «Les hommes s'associent pour trois choses : l'eau, le pâturage et le feu."

\section{La théorie de la complémentarité régionale absolue}

7 Le fleuve constitue une unité régionale que ne peuvent remettre en cause les frontières politiques. Obligation est donc faite aux pays riverains de modifier en rien le cours naturel du fleuve, et ce afin qu'aucun de ces pays n'en ait le monopole. Cette théorie est conforme à l'essence de la jurisprudence islamique Néanmoins, alors que la théorie de la complémentarité régionale absolue englobe le lit du fleuve et les eaux, la loi islamique ne comprend comme bien commun que les eaux. Le lit, lui, reste soumis à ta souveraineté de chaque État riverain et fait partie intégrante de son espace territorial propre.

8 En réalité, la théorie de la complémentarité absolue favorise les pays situés en aval pour ce qui est de la gestion des eaux et ceux situés en amont pour ce qui est de la navigation, alors qu'elle est censée les mettre sur un pied d'égalité.

\section{La théorie de la propriété commune}

9 Les principes de cette dernière s'appuient sur les lois de la nature. En d'autres termes, le fleuve international est propriété commune des États riverains. C'est un cours naturel qui leur octroie, outre les facilités de navigation, les droits et faveurs afférentes : pêche, irrigation et production d'énergie. Par conséquent, aucun des pays riverains n'a autorité pour priver ses voisins de ces droits et faveurs. Don de la nature, le fleuve constitue un bien commun public et un pays n'est en mesure d'exercer ses droits que s'il ne nuit pas à ceux d'un autre État. Cette théorie de la propriété commune s'accorde aux vues et concepts de la jurisprudence islamique. Cependant, et il est intéressant de le noter selon la législation internationale, son application nécessite la signature d'un accord entre les pays concernés, accord inutile dans l'islam. 
10 Les règles d'utilisation des eaux fluviales, si on les étend à l'échelon international, impliquent que les pays riverains en partagent la jouissance conformément à leurs besoins. Plusieurs modes de partage peuvent s'appliquer selon que les pays se font face (auquel cas on peut déterminer pour chacun des jours d'utilisation) ou selon qu'ils sont limitrophes (auquel cas il faut recourir à d'autres moyens de partage plus appropriés, par exemple allouer à chacun un cubage déterminé).

11 Signalons que pour l'essentiel, l'effort des anciens théologiens musulmans a porté sur les divers usages économiques des eaux fluviales, navigation exclue. A telle enseigne qu'ils interprétaient de ce point de vue les versets coraniques relatifs aux fleuves. On peut s'en étonner: l'État islamique n'ignorait pas la navigation fluviale... Qu'on s'en réfère par exemple à la sourate " Les Abeilles », v.15 :

Il a jeté sur la terre des montagnes comme des piliers - afin qu'elle ne branle pas et vous non plus - des rivières, des chemins qui serviront peut-être à vous guider.'

12 Ibn Kuthayr donne de ce verset l'explication suivante: «Dieu a créé des fleuves qui courent d'une région à l'autre, ce qui est une richesse pour les hommes. Leurs sources, situées dans une région, profitent aux populations d'une autre; ils traversent des déserts, des plaines et les terres arides, franchissent des montagnes pour enfin se déverser dans le pays où habitent les hommes auxquels il était destiné ». Et à propos du verset 3 de la sourate «Le Tonnerre » - «C'est lui qui a étendu la terre; il y a placé des montagnes et des fleuves»-, il explique : «Dieu a fait courir des fleuves, des affluents et des sources pour irriguer ce qu'il a créé de fruits de toutes couleurs et formes, d'oiseaux et de senteurs. "

13 Si la théologie classique s'est penchée sur les usages agricoles et ceux qui s'y rattachent, c'est que ces derniers étaient les seuls que l'on connût à l'époque. Cela ne peut donc plus nous être appliqué aujourd'hui. Mais l'on peut dire que la théologie islamique, pour ce qui est de l'élaboration de la théorie de la propriété commune des fleuves internationaux, a devancé les autres législations.

14 Ainsi, Abu Yusuf considère que les gens doivent se désaltérer au grand fleuve (" grand » paraît signifier, traduit en langage contemporain «international») et irriguer leurs terres, et que nul n'a le droit de le leur interdire. Chaque communauté a autorité pour irriguer ses terres, ses palmiers et ses arbres et personne ne peut priver d'eau son semblable. Selon un hadîth du Prophète rapporté par l'imam Ahmad, «Nul ne doit empêcher l'accès à l'eau, de crainte pour les fourrages. »

15 Abu Yusuf poursuit : «Chacun peut, S'il le veut, profiter des eaux du Tigre et de celles de l'Euphrate, et les navires y circuler. Il n'y a pas d'intermédiaire... » Notons ici qu'Abu Yusuf ne fait qu'une rapide allusion à la navigation sur les grands fleuves. Les prescriptions qui précèdent supposent néanmoins un débit d'eau suffisant pour que l'usage souhaité ne souffre d'aucune restriction. Mais que se passe-t-il si le débit se révèle trop faible pour pouvoir couvrir les besoins?

Selon l'auteur de l'ouvrage Al-Ahkâm al-sultâniyya, c'est le premier pays riverain situé en amont qui a priorité pour irriguer ses terres, jusqu'à satisfaction complète de ses besoins. Puis vient le tour du pays voisin, et ainsi de suite jusqu'au dernier. En d'autres termes, la priorité va aux pays situés en amont, ceux de l'aval étant les derniers servis. Il est intéressant de noter que de nombreux spécialistes du droit international contemporain se sont ralliés à cette position. 
17 En ce qui concerne leur usage, les eaux des grands fleuves internationaux sont donc considérées comme bien public commun. L'islam interdit à un État quel qu'il soit de s'approprier pour son seul profit ces richesses naturelles, mais permet à tous les pays d'en bénéficier. Les ressources naturelles en eau relèvent donc du principe de la propriété commune. [...]

18 J'ai souligné plus haut que la relation entre les pays riverains et les eaux fluviales est une relation de droit d'usage, et non de propriété, sauf pour ce qui est du lit du fleuve, possession de l'État concerné. De ce fait, tout pays est en droit de jouir des eaux fluviales qui traversent son territoire. Si, après satisfaction de ses besoins, il subsiste quelque quantité, il doit impérativement la libérer, sans contrepartie, pour le pays qui en a besoin à son tour. Autrement dit, aucun État n'est en droit de refuser le surplus aux autres à partir du moment où cela n'entrave pas son droit. En effet, l'État n'est pas propriétaire des eaux fluviales, qui constituent des ressources naturelles. Il ne dispose que du droit d'usage de ces ressources sur son territoire.

Jouit-il pour autant d'un droit absolu sur la gestion des eaux fluviales? Nullement, car l'État islamique a édicté des règles régissant ce droit. Selon les théologiens, la quantité d'eau que peut retenir un État sur ses terres ne doit pas excéder la hauteur de deux " chevilles ». Une fois ce niveau atteint, il doit libérer le surplus pour le pays suivant, conformément à un hadith du Prophète. Si deux États sont situés à la même latitude en amont du fleuve, ils se partageront les eaux, équitablement si possible. Faute d'entente entre les deux pays, le partage s'effectuera par tirage au sort. Au gagnant reviendra le droit de commencer à irriguer ses terres en fonction de ses besoins. Le perdant puisera à son tour dans le fleuve une quantité d'eau égale. Par souci d'équité, il lui est interdit d'utiliser une quantité supérieure à celle de son voisin. Cet arbitrage ne porte que sur l'organisation du " tour », et non du droit de consommation. Les pays situés en aval ne peuvent bénéficier que du surplus des pays situés en amont. [...]

20 Le hadith du Prophète obéit cependant à certaines considérations temporelles et régionales. Pour cette raison, toute évaluation fondée sur ce hadith ne peut être que de principe, surtout s'il s'agit d'élaborer des normes régissant les relations internationales, et non les relations individuelles. En conséquence, la quantité de réserve sera déterminée par des règles ou des accords, ou selon le principe du moindre préjudice.

21 Il a été mentionné, dans Al-Ahkâm al-sultâniyya, que cette jurisprudence ne s'établit pas, en règle générale, en fonction des conditions de temps et de lieu, mais en fonction des besoins. On distingue cinq cas :

- selon la qualité ou nature de la terre : certaines ne nécessitent qu'une faible quantité d'eau pour leur irrigation, d'autres, au contraire ont des besoins quantitativement élevés ;

- selon la nature des plantations : telle quantité pour les blés, telle autre pour les palmiers et autres arbres ;

- selon les saisons, hiver ou été, à chacune correspondant une quantité d'eau précise ;

- selon la période d'ensemencement : avant ou après les périodes sus-citées, chacun une nécessitant une quantité d'eau définie ;

- selon la nature du fleuve, pérenne saisonnier. En cas d'assèchement on prend les devants pour constituer des réserves. Si le débit est constant, on puise la quantité nécessaire évaluée par les normes internationales ou les traditions.

Ce sont donc les pays situés en amont qui bénéficient des eaux fluviales étant bien entendu qu'ils respectent les principes de bon voisinage et de bonnes intentions et ne 
dépassent pas la quantité déterminée par les pratiques et les normes internationales. Les autres pays riverains limitrophes bénéficient des quantités excédentaires. plus modernes et utilisée à l'aménagement de nouvelles terres (environ 2,5 millions de feddans) ceci indépendamment des eaux de pluie et des nappes phréatiques. La surface des terres arables dans le Sinaï et la région côtière s'en trouverait accrue d'autant. Ces nouvelles méthodes sont déjà appliquées dans la région de la Salihiyya et dans quelques villes nouvelles, mais l'Égypte a un besoin urgent de les généraliser. Elle compenserait ainsi une éventuelle baisse du niveau des eaux, due à des facteurs naturels ou à la construction de barrages susceptibles d'influer sur la quote-part égyptienne des eaux du Nil. Celle-ci a été fixée par la signature du traité de 1959 Si l'Égypte tient à ce que ce traité soit respecté, les autres pays estiment, en revanche, qu'il a été signé pendant l'occupation étrangère dans des conditions qui leur étaient défavorables.

\section{La consommation de l'Éthiopie}

31 L'Ethiopie a été frappée, au milieu des années 80 et au cours des années suivantes, par une grave sécheresse qui a affecté l'agriculture, comme c'est également le cas dans d'autres pays d'Afrique. Cette sécheresse a été causée par l'absence totale ou partielle de pluie. Cette situation a incité les responsables éthiopiens à envisager la construction 
de barrages sur le Nil Bleu, un des affluents les plus importants du fleuve. Israël les a encouragé dans ce sens : l'État hébreu avait en effet tenté d'avoir accès aux eaux du Nil à travers le Sinaï, essayant même d'obtenir pour ce projet l'approbation du président Sadate. Si, à l'époque, l'État hébreu avait obtenu l'aval du chef de l'État, le projet avait été ensuite complètement écarté lorsque Hosni Moubarak a pris le pouvoir en octobre 1981. Israël a alors dirigé ses efforts non plus vers le pays situé à l'embouchure, mais vers le pays situé à la source, et s'est montré prêt à participer à des projets de longue durée visant à assurer une meilleure exploitation des eaux du Nil. Selon certains rapports, Israël aurait effectivement réalisé un relevé aérien en Ethiopie, opération préliminaire à la construction de barrages.

M. Ismail al-Ramli, conseiller de l'ONU pour les questions de l'eau, affirme qu'il est peu probable que l'Ethiopie construise des barrages sur le Nil Bleu, car elle ne pourrait pas en tirer plus d'un demi-milliard de $\mathrm{m}^{3} \mathrm{~d}$ 'eau. Même si elle en tirait 7 milliards de $\mathrm{m}^{3}$. ce qui ne se produirait pas avant cinquante ans, cela n'aurait aucun impact sur la quotepart égyptenne des eaux du Nil, à supposer que des projets hydriques communs soient réalisés. De plus, ajoute-t-il, l'Ethiopie ne dépend des eaux fluviales que pour l'irrigation de 3 millions de feddans; le reste des terres agricoles dépend des eaux de pluie. La surface des terres irriguées par le Nil Bleu n'est que de 20.001 hectares, sans compter les 640 hectares que couvre l'exploitation gouvernementale, le reste étant composé de pâturages et de forêts - cette région est instituée d'un sol rocheux dans lequel les crevasses de basalte absorbent l'e; u qui s'y infiltre. Aussi, si l'on élève de deux mètres le niveau des eaux du lac Tana, comme c'est en projet, pour lui donner un volume de 7 milliards de $\mathrm{m}^{3}$, le surplus des eaux sera englouti par ces crevasses. $\mathrm{Si}$ l'Ethiopie, explique M. al-Ramli défrichait ses forêts (12 millions de feddans) pour transformer cette surface en terres arables, ceci à raison d'un demi-million de feddans par an, elle causerait le plus grand tort aux pays africains voisins en général et aux pays du bassin du Nil en particulier. Le déboisement aurait également pour conséquence une érosion des sols, donc la perte du trésor le plus précieux que possède l'Ethiopie : la terre, c'est-à-dire l'agriculture et la prospérité. Manquer de terre, c'est dépendre de l'étranger et des importations alimentaires en devises lourdes.

\section{Le déboisement}

II est en tout cas certain que la décision de construire des barrages sur le Nil Bleu outre d'autres projets éthiopiens, aura un impact sur les neufs pays du bassin, même si cet impact est limité en ce qui concerne le volume d'eau : car la diminution d'une partie des eaux va entraîner l'aménagement de nouvelles terres agricoles par le défrichage donc la disparition - des forêts couvrant actuellement le bassin du Nil Bleu.

Si une nouvelle sécheresse frappe l'Ethiopie, une partie de sa nombreuse population (42 millions d'habitants) émigrera, en quête d'eau et de nourriture, vers les pays voisins. Il faut donc insister dès maintenant sur les conséquences qu'aurait la construction de barrages : la première victime en serait la population éthiopienne. Un congrès des pays du bassin du Nil serait à cet égard hautement souhaitable, congrès au cours duquel on effectuerait, sous les auspices de l'Organisation de l'environnement de l'ONU, un partage des eaux du Nil, et où l'on renoncerait à un déboisement qui risque d'exposer l'Ethiopie à des sécheresses plus fréquentes et aura des conséquences négatives pour tout le continent. 
congrès pourrait aboutir à la création d'une "Organisation de l'eau » des pays riverains, qui serait chargée de fixer la quote-part des eaux revenant à chacun et d'oeuvrer pour une rationalisation de la consommation d'eau, à l'instar de l'Organisation du bassin du Zambèze en Afrique. [...]

Il revient au gouvernement égyptien d'examiner rapidement ces projets et de mettre en lumière leurs conséquences immédiates et futures. [...] L'Égypte devrait entreprendre dès maintenant de rationaliser sa consommation d'eau: restreindre la consommation individuelle pour qu'elle dépasse pas le niveau de celle de l'Europe, ou même soit moindre que celle-ci : favoriser chez les citoyens une prise de conscience de la valeur de l'eau en généralisant les méthodes d'irrigation scientifiques et en abandonnant la méthode traditionnelle; souligner les dangers du gaspillage. Ces mesures permettraient de prévenir tout danger futur, qu'il soit d'origine naturelle ou humaine, et de veiller avec vigilance sur tout ce qui peut porter atteinte au Nil, source de vie de l'Égypte.

\section{Traduit de l'arabe par Muriel Berset}

\section{Israël : des besoins croissants en eau}

\section{Al-Charq al-Awsat, 4 août 1990}

Depuis la création de l'État d'Israël, ses dirigeants ont [...] mis en application deux moyens pour garantir au pays la quantité d'eau nécessaire, que l'on estime à environ 920 millions de $\mathrm{m}^{3}$ :

- 380 millions de $\mathrm{m}^{3}$ obtenus grâce à divers projets d'exploitation des sources et des nappes d'eaux souterraines existant sur le territoire ;

- 540 millions de $\mathrm{m}^{3}$ obtenus par le détournement des eaux du Jourdain de Jisr Banat Yaqub au sud du désert du Néguev.

Bien que ce dernier projet ait été réalisé en 1964, Israël s'est à nouveau trouvé confronté à l'insuffisance de l'eau. Cette crise s'est trouvée partiellement résolue par la guerre de 1967, du fait de l'occupation de nouvelles terres arabes et de l'exploitation des ressources hydrauliques offertes par les régions occupées, Mais avec l'augmentation de la population et l'expansion de nouvelles terres à irriguer, la crise est réapparue. Actuellement et depuis les années 70, Israël utilise plus de $95 \%$ de la totalité de l'eau dont il dispose. Cette exploitation intensive des ressources hydriques, et en particulier des eaux souterraines, a provoqué des conséquences néfastes, dont principalement l'augmentation de la salinité des puits, ce qui les rend inutilisables à de nombreux égards. Certains puits se sont d'ailleurs asséchés depuis le printemps 1984.

Différentes études confirment que les besoins croissants d'Israël en eau ne sont pas uniquement attribuables au développement de l'agriculture, mais aussi à l'augmentation de la consommation domestique, parallèle à la croissance démographique: la population israélienne s'élève aujourd'hui à 4.5 millions d'habitants, et ne tardera pas à en atteindre 5,2 millions avec l'immigration des juifs en provenance d'Union soviétique, des pays de l'Est et d'Afrique Noire (un demi-million d'immigrants sont attendus pour les trois années à venir). L'État hébreu devra par conséquent fournir, ne serait-ce qu'en raison de cet accroissement, environ 268,5 millions de $\mathrm{m}^{3}$ d'eau supplémentaires en 1995. 
Selon diverses estimations, le manque d'eau s'élèvera environ, en l'an 2000, à 800 millions de $\mathrm{m}^{3}$; Israël consommant plus de $95 \%$ des ressources hydriques, comment vat-il couvrir ses besoins? Question d'autant plus pressante que l'arrivée d'émigrés soviétiques n'avait pas été calculée dans les estimations.

\section{La technologie ou la guerre}

Israël a le choix entre deux solutions :

- L'emploi de technologies modernes, parmi lesquelles. Israël a déjà utilisé :

- La réduction de l'évaporation du lac de Tibériade (laquelle entraine une perte de l'ordre de 300 millions de $\mathrm{m}^{3} / \mathrm{an}$. La technique utilisée consiste à recouvrir la surface de l'eau d'une mince pellicule d'huile qui empêche l'eau de s'évaporer. Les Israéliens reconnaissent l'échec total de cette expérience, le vent provoquant des vagues qui brisent la couche d'huile.

- La provocation de pluies artificielles par l'injection, dans les nuages, de produits tels que le bioxyde de carbone. L'expérience a coûté un demi-million de dollars. De plus, il est difficile, sinon impossible, de déterminer où tomberont les pluies en Galilée. Même si l'on parvenait à la perfectionner, cette technique ne pourrait donner que de faibles résultats.

- Le dessalement, procédé utilisé pour Eilat depuis les années 70 et qui satisfait $50 \%$ des besoins domestiques de cette ville, La station de dessalement qui produit un million de gallons d'eau annuellement, ne constitue que la première étape d'un plan de dessalement entamé en 1988 et échelonné sur une période de 15 ans. Dans une deuxième étape, l'on mettra en fonctionnement une station américano-israélienne chargée de produire 12 millions de gallons et employant des sources d'énergie non-nucléaire. Dans une troisième étape, il est prévu de construire sur la côte méditerranéenne une station nucléaire devant produire 120 millions de $\mathrm{m}^{3} /$ an à un faible coût de production : le mètre cube d'eau reviendrait à 20 à 30 cents. Toutefois, le prix effectif dépassera largement cette estimation, compte tenu de l'inflation. Le prix de revient du dessalement de l'eau de mer reste très élevé et ne peut constituer qu'une solution partielle.

- Le recyclage des eaux usées, procédé utilisé depuis la fin des années 70 et qui fournit 30 millions de $\mathrm{m}^{3}$ /an. D'après le Jerusalem Post du 27 juillet 1978, on prévoyait que cette quantité s'élèverait, à partir de 1986, à 100 millions de $\mathrm{m}^{3}$ /an et servirait à irriguer le désert du Néguev. Une nouvelle station, de recyclage doit être créée, ce qui élèverait la quantité d'eau fournie à 158 millions de $\mathrm{m}^{3} / \mathrm{an}$. Mais l'eau obtenue par ce procédé ne dépassera pas 325 millions de $\mathrm{m}^{3}$ jusqu'à l'an 2000.

- L'irrigation au goutte à goutte, qui constitue une économie importante dans la consommation de l'eau (environ 50\%) mais s'avère plus coûteuse que l'irrigation par arrosage, et se montre peu pratique sur de grandes surfaces, l'entretien des appareils nécessitant une main-d'œuvre importante.

L'augmentation des ressources hydrauliques par la mise en œuvre de technologies modernes n'a donc donné que des résultats limités. Reste l'autre solution:

- exploiter les ressources hydrauliques non locales pour satisfaire les besoins de la Cisjordanie. Telle est la solution qui transparaît à travers les propos du président du conseil d'administration de la compagnie Tahal, publiés dans le quotidien Davar du 26 novembre 1978. Quelles sont ces ressources? 


\section{Le Jourdain}

45

[...] Le conflit à propos des eaux du Jourdain a commencé en 1953, lorsqu'Israël a entamé les travaux de détournement des eaux du fleuve qui devaient s'effectuer en sept ans. Le 2 juilet 1953, la Syrie a intercepté les premières opérations israéliennes de creusement du canal principal dans la zone de désarmement située près de la frontière syrit nne (au nord de Jisr Banal Ya'qub). Damas a porté plainte en espérant que le conseil de sécurité adopterait une résolution rapide. [...]

Le président Eisenhower a alors chargé le conseiller Eric Johnston de le représenter dans les négociations entre les parties en conflit. Il s'agissait de parvenir à un plan commun d'exploitation des eaux du Jourdain.

Soutenu par la diplomatie américaine, le plan Johnston prévoyait :

- au-dessus du Hasbani, un barrage devant constituer une source d'énergie et permettre l'irrigation des terres de Galilée ;

- au-dessus des fleuves Banias et Tall al-Qadi (Atwan), deux barrages, et l'acheminement, à travers de longs canaux, des eaux du Hasbani ayant alimenté la centrale électrique, pour qu'elles irriguent la région de Hawla, les collines de Haute-Galilée et la vallée de Marj Ibn 'Amir dans les territoires occupés ;

- au-dessus du Yarmouk, à Maqarîn, un barrage d'une capacité de 175 millions de $\mathrm{m}^{3}$, destiné à la production l'énergie ;

- au-dessus du Yarmouk également, à al-'Adasiyya, un autre barrage destiné à conduire les eaux de la crue vers le lac de Tibériade et vers la région située à l'est des Agwar ;

- à l'est et à l'ouest du Jourdain la création de deux canaux devant permettre l'irrigation des terres riveraines (région d'al-Agwar, entre le Yarmouk et la Mer Morte) ;

- divers barrages et canaux destines à recueillir l'eau des ruisseaux de la vallée et celle des crues, sur les rives du Jourdain, pour irriguer le bassin :

- renforcement des rives du lac Tibériade de façon à augmenter la capacité de réserve et le niveau des eaux (ce dernier pouvant alors être plus élevé de deux mètres).

Le rapport Johnston estimait à 1.213 millions de $\mathrm{m}^{3}$ la quantité totale de l'eau dans le bassin du Jourdain et proposait de la répartir comme suit :

- 774 millions de $\mathrm{m}^{3}$ pour la Jordanie ;

- 45 millions pour la Syrie ;

- 394 millions pour Israël.

Les pays arabes s'y sont opposés, objectant que la quantité et la qualité d'eau qui leur était impartie était insatisfaisante. De plus, [...] ils se trouveraient à la merci d'Israël. Leur propre rapport insistait sur le principe selon lequel l'exploitation des eaux du fleuve devait se limiter à la région de son bassin [...]. Au fil des négociations entamées autour du plan Johnston, le conflit s'est apaisé : les pays arabes ont admis l'exploitation de l'eau hors du bassin du fleuve, et de son côté, Israël a renoncé à rattacher le Litani au système du Jourdain. Les désaccords ont cependant persisté sur d'autres aspects du problème. Les Israéliens ont notamment refusé la proposition arabe visant à considérer le lac de Tibériade comme une réserve commune, où chacun pourrait puiser selon ses besoins. D'autre part, les Arabes exigeaient que la répartition de l'eau soit soumise à un contrôle international, ce qu'Israël refusait. Johnston proposa alors un "plan commun » garantissant à Israël 400 millions de $\mathrm{m}^{3}$ d'eau. Mais cette fois, alors que le comité technique arabe acceptait ce plan, c'est la Ligue arabe qui s'y opposait, objectant qu'il avait des visées politiques. Bien que le conseil des ministres israélien n'eût pris 
aucune décision, Israël et la Jordanie convinrent, officieusement, de respecter les taux attribués à chacun et de commencer à mettre en œuvre leurs projets respectifs : pour Israël, il s'agissait du pipeline national, pour la Jordanie, du canal de Jawr.

Le conflit allait resurgir avant l'achèvement du pipeline, en 1964. A cette date se réunissait le sommet arabe destiné à traiter du détournement des eaux du Jourdain. Le 13 janvier 1964, le sommet émettait ses résolutions : il recommandait la création d'une organisation chargée de l'exploitation des eaux du Jourdain et la mise en place des projets nécessaires à la gestion des ressources hydrauliques arabes. Il s'agissait de mettre un coup d'arrêt aux visées expansionnistes d'Israël en évitant une confrontation militaire. Les pays arabes pouvaient opter pour une des deux solutions suivantes :

- acheminer les eaux du Hasbani vers le Litani, et celles du Banias vers le Yarmouk;

- ou acheminer les eaux du Hasbani et du Banias vers le Yarmouk. C'est la .première solution qui prévalut. En outre, on projetait la construction du barrage de Makhiaba. Le coût, d'environ 200 millions de dollars, devait être partiellement pris en charge par l'Arabie Saoudite et l'Égypte. Deux conséquences importantes devaient résulter de l'exécution du projet ;

- les Arabes récupéreraient 250 à 300 millions de $\mathrm{m}^{3}$ de leurs ressources hydrauliques ;

- en ré-orientant les affluents Jourdain, on empêcherait l'apport d'eau douce au lac de Tibériade, ce qui en augmenterait le taux de salinité et diminuerait la qualité pour l'exploitation, le détournement du Jourdain deviendrait ainsi caduc.

\section{La guerre de 1967}

51 Les pays arabes commencèrent la construction du barrage de Makhiaba (barrage Khatid ibn al-Walid) qui comportait pour Israël les conséquences les plus graves puisqu'il empêchait, comme on l'a dit plus haut, l'eau douce des affluents du Jourdain de parvenir au lac de Tibériade. L'État hébreu annonça qu'il ne resterait pas passif. Il considérait la construction du barrage de Makhiaba comme un acte d'agression, puisqu'il portait atteinte à ses ressources hydrauliques. Il menaça donc les pays arabes de représailles, et ces menaces ne restèrent pas lettre morte: elles furent suives de nombreux raids aériens visant les chantiers de construction du barrage. Ces attaques allaient atteindre leur apogée en avril 1967, et s'étendre à la Syrie [...].

La guerre de 1967 eut des conséquences importantes sur le plan des ressources hydrauliques, du fait de l'occupation du Golan et de la rive ouest du Jourdain : Israël en tirait le plus grand profit. L'occupation du Golan rendait impossible aux pays arabes la poursuite des travaux entrepris, puisque Israël occupait à présent la moitié de la longueur du Yarmouk (contre $10 \mathrm{~km}$ seulement avant la guerre). Avant le conflit, 20\% du projet du Yarmouk avaient été réalisés, mais avec le début des hostilités, les travaux de construction du barrage de Makhiaba, qui devait retenir 200 millions de $\mathrm{m}^{3} \mathrm{~d}$ 'eau, furent totalement interrompus. Ce fut également le cas du barrage de Maqarîn, qui devait contenir 350 millions de $\mathrm{m}^{3}$ d'eau ; Israël résolvait ses problèmes hydrauliques aux dépens des régions arabes occupées.

Avec la relance du conflit à propos du Jourdain, il semble presque impossible d'imaginer, dans la nouvelle situation politique, un projet de développement commun des ressources hydrauliques dans la région. Le seul projet dont on peut concevoir la réalisation est celui du développement du Yarmouk entre la Jordanie et la Syrie. Ce fleuve commun aux deux pays constituait, sur une longueur de $40 \mathrm{~km}$, la frontière entre 
la $\mathrm{S}^{\wedge}$ rie et la Jordanie avant de devenir la limite (sur $10 \mathrm{~km}$ ) séparant la Jordanie d'Israël avec l'occupation du Golan. Le débit total de ce fleuve ne dépasse pas 500 millions de $\mathrm{m}^{3}$ /an. En 1952, un expert américain avait proposé de construire un barrage au-dessus de ce fleuve, dans la région de Maqarîn; le projet fut interrompu par la relance du conflit concernant le détournement du Jourdain et l'explosion de la guerre de 1967.

La Jordanie a cependant repris l'idée de construire ce barrage dans le cadre de son plan septennal de 1975-1982. Entre 1975 et 1980 se sont succédées plusieurs étapes préliminaires : analyses, études de terrain, documentation, appels d'offre... Pour ce qui est du financement, le projet a reçu des appuis étrangers, dont celui de l'administration Carter. Le rapprochement entre la Syrie et la Jordanie a abouti à la signature, en septembre 1987, d'un accord en vue de la construction de ce barrage, dès lors nommé «Barrage de l'Unité » (al-Wihda). Dans la nouvelle perspective, on envisageait d'élever le barrage à une hauteur de 100 pieds et de diminuer sa capacité de réserve à 220 millions de $\mathrm{m}^{3}$. C'est à la Jordanie que serait attribuée la plus grande quantité d'eau mais, en revanche, c'est à la Syrie que reviendrait la part d'énergie électrique la plus importante. Encore une fois, Israël fit obstacle à l'exécution de ce projet : trop exposé à la présence militaire des Israéliens sur les hauteurs du Golan, il fut abandonné au profit de celui de Maqarîn, plus éloigné de l'armée d'occupation.

Israël, avec l'implantation de colonies en Cisjordanie, allait, de plus, exiger une augmentation de sa part du Yarmouk [...]. De plus, Menahem Béguin demandait au président américain d'alors, Jimmy Carter, des garanties contre le danger que le barrage de Maqarîn pouvait représenter pour les eaux du Yarmouk, dans le triangle avoisinant les territoires occupés. Israël demandait également que l'eau réservée à l'irrigation du triangle en question passe de 17 millions à 40 millions $\mathrm{de}^{3}$, et exigeait enfin 140 millions de $\mathrm{m}^{3}$ supplémentaires sous prétexte de pouvoir augmenter le débit du Jourdain et exploiter ses eaux pour l'irrigation de la rive ouest. L'envoyé américain Philip Habib tenta de parvenir à un accord autour du projet de Maqarîn mais la crise libanaise mit fin à ses efforts.

Israël n'a Jamais cessé de s'opposer à la construction du barrage du Yarmouk. Au contraire, il a exploité sa nouvelle position, après 1967, pour faire pression sur la Jordanie. De son côté, la Jordanie se méfie des pratiques d'Israël depuis son occupation de la rive nord du Yarmouk : entre autres, nombreuses attaques contre le canal al-Jawr depuis 1968. On sait également qu'Israël cherche à entraver le cours du fleuve. La médiation des États-Unis a donc été nécessaire pour que la Jordanie accepte que soient entrepris des travaux de purification du bassin. Les Israéliens ont commencé à détourner les eaux du Yarmouk afin d'en augmenter le débit en direction du lac de Tibériade. Selon les estimations jordaniennes, Israël en retire 100 millions de $\mathrm{m}^{3}$ alors que la part qui lui est attribuée, d'après le plan Johnston, ne devrait pas dépasser 25 millions de $\mathrm{m}^{3}$ (Selon des sources israéliennes datant d'avril 1984, Israël avait l'intention de prélever 60 à 70 millions de $\mathrm{m}^{3} \mathrm{du}$ Yarmouk chaque année. Tous ces facteurs ont incité la Syrie à construire de nombreux petits barrages destinés à retenir l'eau des vallées et des sources, ce qui a en pour effet de diminuer de 400 à 220 millions de $\mathrm{m}^{3} / \mathrm{an}$ la quantité d'eau s'acheminant vers la Jordanie. Devant cette situation critique, cette dernière a proposé de s'entendre avec l'Irak pour l'acheminement d'eau de l'Euphrate vers la Jordanie, au moyen de pipelines. Cependant, à cause de la nature du terrain, du faible rendement du projet et de sa difficulté de financement, cette idée a 
été abandonnée. La Jordanie s'est donc vue dans l'obligation de renouer, en 1987, avec la Syrie pour reprendre le projet du barrage de Maqarîn. Cette fois, il devait être réalisé dans de nouvelles conditions, les projets que la Syrie avait menés à bien de son côté ayant eu pour effet de diminuer la capacité de réserve du barrage passée de $500 \mathrm{~m}^{3}$ (dans les années 60 à 70 à 220 millions en 1987.

La situation a de nouveau changé lorsque la Turquie a décidé de retenir, durant un mois entier, les eaux de l'Euphrate, les empêchant de parvenir en Syrie. La nouvelle situation de la Syrie, son besoin d'eau urgent pourrait bien modifier son intention de partager les eaux du Yarmuk avec la Jordanie. La situation de la Syrie est rendue plus critique encore par l'attitude d'Israël, qui continue de faire obstacle au projet de barrage de Maqarîn. Selon les déclarations d'un porte-parole israélien, le 17 décembre 1987, Israël aurait demandé aux États-Unis d'intervenir pour en obtenir la suspension : le barrage de Maqarîn, en effet, priverait l'État hébreu des milliers de mètres cubes d'eau qu'il tire du Yarmouk.

Traduit de l'arabe par Madiha Doss.

\section{Turquie : une inépuisable réserve d'eau}

Joseph R. Gregory

World Monitor, 28 novembre 1991

Selon un proverbe musulman, "à chaque gorgée d'eau qu'il prend, l'oiseau lève les yeux au ciel en signe de gratitude ». Cependant, comme tous les dons terrestres, l'eau a été inégalement répartie. Ainsi, en Turquie, le grand nombre de voies navigables n'empêche pas l'existence de vastes zones arides, et un bon nombre des 56 millions d'habitants que compte le pays souffrent du manque d'eau. Les réserves dont ils disposent suffisent à peine à leurs besoins en eau potable. Aussi est-il d'usage en Turquie, lorsque vous sortez tout frais d'un bain, de vous entendre dire : "Sihhat ler olsun » (« Puisse cette fraîcheur durer plusieurs heures »).

Paroles de courtoisie qui évoquent la vie raffinée des sultans.

C'est cependant au cours des deux semaines passées sous le soleil torride des plaines de Harran-Urfa,„à la frontière sud-est du pays, que j'ai réalisé que ces mots étaient bien autre chose qu'une expression de politesse orientale. Dans cette région, en effet, vous ne prenez un bain que si l'occasion s'en présente. Lorsque cela m'est arrivé - et malgré la sueur qui me couvrait déjà, à peine rhabillé - ma journée en était transformée.

Dans un an, grâce au projet de développement de l'Anatolie du sud-est, prendre un bain ne sera plus un luxe. Ce projet, le GAP (Guneydogu Anadolu Projesi) a été conçu dans le but d'alimenter une des régions les plus pauvres du pays en eau d'irrigation et en énergie hydraulique. Vers la fin de 1992, si tout se passe comme prévu, l'eau du réservoir situé derrière le nouveau barrage Atatürk commencera à se déverser dans les deux canaux qui la répartiront dans toute la campagne qui entoure Sanliurfa, l'une des plus grandes villes de la région, à travers un vaste réseau d'irrigation. Davantage d'eau, donc davantage de soja, de coton et autres produits agricoles qui feront de la plaine de Harran-Urfa un véritable grenier. Les habitants de la région se plaisent à dire que, grâce au GAP, la terre redeviendra aussi verte qu'elle l'était du temps où, selon la légende, Adam et Eve, chassés du paradis, étaient venus y planter un nouvel Eden. 

visant une paix globale dans la région. Le fait que l'eau devienne plus précieuse que le pétrole a amené les gouvernements les moins stables à prendre conscience de leur intérêt à chercher ensemble des solutions à leurs problèmes, Comme le dit Inan, « un pays auquel on coupe le pétrole peut tenir pendant des mois, voire des années. Mais privé d'eau, un pays ne peut pas survivre une semaine. » l'eau au Moyen-Orient, à l'initiative d'un groupe de recherche à but non lucratif établi à Washington (le GWSI - Global Water Summit Initiative). Le président George Bush, les 
chefs d'État des pays de la Communauté européenne, les dirigeants japonais et soviétiques ainsi que ceux des pays du Moyen-Orient y ont été invités pour discuter des problèmes régionaux relatifs à l'exploitation de l'eau et du soutien à apporter aux projets hydrauliques. En dépit des réserves formulées ci-dessus, la plupart des pays arabes ont annoncé qu'ils participeraient à cette conférence. [...]

71 Israël et l'Irak ont été exclus du projet du pipe-line de la paix, projet qui ne constitue qu'un point de l'ordre du jour de cette conférence et n'en est pas le plus important. M. Inan, et avec lui d'autres partisans du projet, soutiennent cependant que ce projet pourrait constituer un test prouvant que la coopération économique peut ouvrir la voie à la paix politique. D'autres, en revanche, estiment que les problèmes économiques de la région ne pourront être résolus que si les divergences politiques sont réglées. Par où faut-il donc commencer? La coopération économique ou la paix politique ? Certains affirment que les Turcs possèdent suffisamment de réserves en eau et qu'ils ont à la fois la compétence technique et des raisons économiques de vouloir prendre en charge la répartition des eaux dans la région. En contrepartie, les pays arabes voisins aideraient au financement du projet par la construction d'oléoducs vers l'Europe, lesquels passeraient par l'Anatolie, région pauvre en ressources pétrolières.

Il n'en reste pas moins que, sans volonté politique de coopérer, ce pipe-line pour la paix ne verra jamais le jour. Si tous doivent «tirer l'eau du puits ", les nations participant à ce projet devront s'engager à ne pas utiliser te pipe-line comme moyen de pression pour régler d'autres différents. Or, les querelles pour l'eau sont malheureusement liées à d'autres rivalités... Le mot « rival » lui-même ne vient-il pas du latin rivalis qui signifie " riverains tirant leur eau du même cours »? Aujourd'hui, la Turquie, la Syrie et l'Irak mènent une lutte acharnée pour la «riveraineté » sur le Tigre et l'Euphrate. Ces deux fleuves sont en effet la principale source d'eau aussi bien pour le projet turc connu sous le nom du GAP, que pour la Syrie et l'Irak. Ces deux derniers reprochent au GAP de retenir l'eau qui leur revient de droit, chose que les Turcs nient catégoriquement.

Quelle que soit la vérité, ces querelles montrent bien les difficultés soulevées par le partage des eaux entre deux pays. Qu'adviendra-t-il lorsque se trouveront engagés les intérêts d'une demi-douzaine de nations entre lesquelles les tensions remontent bien loin dans l'histoire? Il n'est guère étonnant que, dans un tel contexte, les plus cyniques aient surnommé ce projet le « pipe-rêve » de la paix....

La nature a voulu que les Turcs n'aient pas à partager avec quiconque les eaux du Ceyhan et du Seyhan. En effet, ces deux grands fleuves, alimentés par les pluies et la fonte des neiges, déversent chaque jour 39 millions de $\mathrm{m}^{3}$ d'eau (environ 10,3 milliards de gallons) dans la Méditerranée.

75 En 1987, les Turcs ont confié à Brown \& Root, société d'ingénierie américaine, l'étude de faisabilité du projet Le coût de cette étude s'élevant à 2,7 millions \$, Ankara s'engageait à en payer 800.000 et Washington 1,2 million tandis que la Grande-Bretagne acceptait d'en verser 700.000 , à condition toutefois que les pays concernés montrent leur volonté de coopérer.

76 Il est prévu que les Turcs acheminent 6 millions de $\mathrm{m}^{3}$ d'eau (environ 1,6 milliards de gallons) par jour vers les deux principaux pipe-lines. Financièrement, c'est de la contribution de l'Arabie Saoudite et du Koweït que dépend essentiellement la réalisation du projet. Or ni l'un ni l'autre ne font preuve d'enthousiasme. Officiellement, les Turcs attribuent la réticence des Arabes aux sommes importantes que ces derniers ont déjà investies dans les installations de dessalement de l'eau de 
mer. Ils ajoutent que, cependant, la vulnérabilité de ces installations a été largement démontrée lors de la guerre du Golfe : la pollution ne les a pas épargnées. Par ailleurs, disent-ils, « le projet vise à augmenter les réserves d'eau existant en Arabie Saoudite, non à les remplacer, et le coût de l'eau «importée " sera nettement moins élevé - le tiers du prix - que celui de l'eau de mer dessalée », selon M. Inan.

De leur côté, plusieurs Arabes maintiennent que le dessalement est moins onéreux. Selon les analystes, aucune étude comparative fiable des coûts n'a été réalisée dans ce domaine jusqu'à ce jour. Mais quel que soit le coût réel de l'opération, plusieurs personnalités arabes semblent animées des mêmes sentiments que ce diplomate arabe qui a demandé à garder l'anonymat et qui déclarait à l'Agence France Presse: "Je préfère payer plus cher que livrer ma tête au bourreau ». De son côté, Eric Rouleau, ancien correspondant du Monde qui a vécu une trentaine d'années au Moyen-Orient, affirme que "sans l'appui de l'Arabie Saoudite, le succès du projet turc est menacé. officiellement, l'Arabie prétend ne pas avoir besoin d'eau; en réalité, c'est d'une opposition politique qu'il s'agit. »E. Rouleau reconnaît que la résolution du problème de l'eau constitue sûrement une incitation à la paix dans la région, mais, précise-t-il, ce n'est qu'un problème parmi d'autres : «Le pipe-line de la paix ne pourra être construit que si la région est bien gouvernée et qu'il y règne une paix véritable ».

78 Nacati Utkan, ambassadeur de Turquie en Irak, soutient le point de vue opposé : « Nous devons, dit-il, briser ce cercle de méfiance». Rappelé de Bagdad à la rupture des relations diplomatiques avec l'Irak lors de la guerre du Golfe. N. Utkan ne voit dans le pipe-line de la paix qu'un exemple parmi d'autres des projets qui pourraient favoriser la paix dans la région. Certains experts considèrent aujourd'hui les $\mathrm{CMB}$ - Confidence Building Measures («Mesures pour l'instauration de la confiance») - comme le premier pas sur le chemin de la paix. Il faudrait pourtant, dit-il, commencer par les CBP - Confidence Building Projects (« Projets pour l'instauration de la confiance»), «Une fois prouvée leur fiabilité, ajoute-t-il, nous pourrons espérer une coopération plus solide et une paix durable. »

Côté syrien, l'on soutient que le projet de pipe-line n'a d'autre but que d'étouffer les craintes quant à la perte des eaux du Tigre et de l'Euphrate que risque de provoquer le GAP. Autrefois, ces deux fleuves titanesques ont nourri la civilisation occidentale dans le fertile croissant de la Mésopotamie. Aujourd'hui, la Turquie exploite leur puissance pour construire, d'ici l'an 2000, 17 centres hydro-électriques et 21 barrages à travers le sud-est de l'Anatolie. Le GAP, qui couvre environ $10 \%$ du territoire turc, est censé augmenter du 1/3 l'énergie hydro-électrique de ce pays.

D'après une étude menée par Thomas Naff, de l'université de Pennsylvanie, le GAP, une fois achevé, pourrait détourner une grande quantité de l'eau de l'Euphrate au profit de l'irrigation et de l'électricité en Turquie, ceci aux dépens de la Syrie qui y perdrait $40 \%$ de sa part, et de l'Irak, qui y perdrait $80 \%$ de sa part. Pourtant, aucune loi internationale ne règle la répartition des droits des pays riverains sur le Tigre et l'Euphrate. Selon les Turcs, pareille loi n'est pas nécessaire : «On ne prive personne d'eau, pas même son ennemi », déclare Kamran Inan. « La Syrie et l'Irak ne manqueront jamais d'eau », précise-t-il.

81 Cependant, Syrien et Irakiens craignent d'être les derniers servis en cas de pénurie. Le gouvernement turc n'a-t-il pas déjà, en janvier 1990, été amené à réduire le flux de l'Euphrate durant 30 jours pour remplir le réservoir de l'énorme barrage Atatürk ? La Syrie et l'Irak avaient alors âprement protesté. La Syrie s'était ensuite apaisée lorsqu'à 
la suite de l'invasion du Koweït par l'Irak, Ankara et Damas avaient pris position contre Bagdad aux côtés des Alliés. Mais le conflit n'est pas terminé pour autant. Pour les Turcs, il est plus logique de construire des réservoirs en Turquie, où le phénomène d'évaporation est moins important. Par ailleurs, le contrôle du niveau de l'eau effectué en un point plus proche des sources du fleuve permet de mieux maîtriser les inondations et de protéger les trois pays de sécheresses telles que celle qui avait sévi en 1989. D'après K. Inan, la Turquie, pour aider ses voisins, avait augmenté le débit en ralentissant le remplissage de son réservoir, provoquant un retard sur le calendrier du GAP.

En fait, là où les Turcs voient un accord reposant sur l'honneur, Syriens et Irakiens ne voient qu'un rapport de force. C'est ce qu'affirme Eric Rouleau en ces termes : « Rien ne leur garantit que la Turquie continuera à leur fournir l'eau à l'avenir.» Il n'existe en effet aucune convention internationale à ce sujet. Ni la Cour internationale de Justice ni les Nations Unies ne peuvent donc leur venir en aide. Ils sont livrés au bon vouloir des Turcs...

Le prophète Muhammad avait prédit qu'un jour on trouverait de l'or dans l'Euphrate. D'après Ibrahim Halil Celik, c'est le GAP qui fera de cette prophétie une réalité.

I. H. Celik est le maire de Sanliurta, qui compte 290.000 habitants et se trouve à environ 35 miles au nord de la frontière syrienne. Selon la légende, c'est dans cette ville qu'est né le prophète Abraham. On dit aussi qu'elle était prospère jusqu'au jour où, il y a six siècles, elle fut envahie par les Mongols qui dévastèrent arbres et plantes et asséchèrent les ruisseaux. Toutefois, ni les historiens ni les géographes n'ont pu déterminer la cause de cet assèchement.

Dans cette région, le premier devoir qu'impose l'hospitalité envers un visiteur, c'est de lui offrir à boire. Bien installé dans son bureau spacieux et climatisé, I. H. Celik me prie donc, ainsi que l'interprète qui m'accompagne, de lui faire l'honneur de boire quelque chose. En attendant il discute, avec une demi-douzaine de ses amis, de l'étemel problème de sa ville : une fois de plus, les pompes d'eau sont en panne,

Un des avantages du GAP, et non des moindres, sera justement d'augmenter la pression de l'eau. Bien que tousceux que j'ai rencontrés en Turquie soient favorables au projet, chacun semble pourtant y trouver quelque chose à redire. Ainsi, les sociologues s'inquiètent du relogement de milliers de personnes qui vivaient dans les quelque 250 villages inondés par les réservoirs du barrage ; les archéologues contestent l'inondation de plus de 200 sites avant qu'ils aient fait l'objet d'une étude complète et sérieuse ; les agronomes mettent en garde contre le fait que, si le système d'irrigation n'est pas réglé avec le plus grand soin, l'accumulation de sel pourra ruiner le sol.

87 Pour sa part, I. H. Celik craint que les changements amenés par le GAP n'ébranlent la culture locale. "Si les gens n'utilisent pas l'eau avec la plus grande sagesse, ils s'exposeront à maints problèmes, dit-il. Ils oublieront l'importance de l'Islam... »

88 I. H. Celik est le leader régional du parti fondamentaliste "Refah », dont le nom signifie "prospérité ». Bien que favorable au GAP, ce parti conteste la politique adoptée par le gouvernement OzaI en ce qui concerne le ralliement de la Turquie aux États-Unis et sa demande d'adhésion à la Communauté européenne. Ce parti préférerait que la Turquie oeuvre plutôt en faveur d'une communauté économique avec les pays arabes, sur le modèle de la Communauté européenne mais dans le respect des préceptes de l'Islam. Or, d'après I. H. Celik, cet objectif est menacé par le pipe-fine de la paix, et le projet 
proposé par Ozal n'est en fait «qu'une partie du complot israélo-américain pour contrôler le Moyen-Orient ».

Mais ce pipe-line de la paix ne sera-t-il, pas utile aux peuples musulmans?

A cette question, Celik répond qu'il servira avant tout à donner à Israël l'eau de la Turquie. C'est ce qui se produira, dit-il, car « ce sont les juifs qui mènent Washington et ses alliés. Les États-Unis ne veulent pas la paix. Ils ont prouvé qu'ils étaient « une nation de sang et de larmes » en bombardant les civils à Bagdad durant la guerre du Golfe. Maintenant que la guerre froide a pris fin, l'OTAN va se transformer en une organisation anti-islamique... »

Notre interprète, un jeune homme d'une vingtaine d'années, diplômé de l'université, est contrarié par les propos du maire: «Je suis musulman, dit-il en anglais. L'islam n'est pas une religion de violence mais d'amour... »

Tout comme dans la réponse de la Turquie à Frank Rizzo, l'impétueux ex-maire de Philadelphie, les paroles de Celik trahissent ses sentiments, mais dans un style plus ironique. Interrogé sur la raison pour laquelle un homme qui dit vouloir la paix a sur son bureau un briquet en forme de grenade, il répond que c'est un souvenir de son voyage en Amérique...

A son tour, il demande pourquoi les Américains se soucient-ils tant de la pénurie d'eau au Moyen-Orient. En réponse, je lui rappelle une anecdote sur Mustafa Kemal, le futur Atatürk, père de la Turquie contemporaine. Lors de la révolution turque, à l'issue de la première guerre mondiale, l'Union soviétique, espérant convertir Kemal au communisme, l'avait invité à une fête organisée à l'ambassade. Constatant que les diplomates du nouvel état "égalitaire » avaient toutes les boissons qu'ils souhaitaient alors qu'il était formellement interdit aux serviteurs d'y goûter, Kemal demanda que les serviteurs aient, eux aussi, à boire. A l'objection formulée par un commissaire, Kemal cita un proverbe turc qui s'est avéré prophétique dans le cas de l'Union soviétique et qui s'appliquera peut-être un jour au Moyen-Orient: "Le jour où un homme boira alors qu'un autre ne pourra que le regarder faire, ce sera le signe précurseur de la fin des temps ».

Traduit de l'anglais par Samia Rizq.

\title{
Ressources hydrologiques de la Jordanie
}

\author{
Ali Z. Ghezawi \\ Jordan Times, 15-16 août 1991 \\ En l'an 2005, les besoins en eau de la Jordanie augmenteront de plus de 65\%, et sa \\ population de plus de $70 \%$. Le présent article se propose de faire la lumière sur la \\ question des ressources en eau de ce pays, et d'en tirer les recommandations qui \\ s'imposent aux plans local et régional. [...] En dépit de leur relative rareté, tes \\ ressources naturelles constituent un élément majeur de l'économie nationale \\ jordanienne. Et l'eau fait partie des maigres ressources de la Jordanie, qui compte \\ surtout sur la pluviosité pour couvrir sa consommation domestique, agricole et \\ industrielle.
}


Ces quatre dernières années, la moyenne des précipitations annuelles en Jordanie a été d'environ 9,19 millions de mètres cubes $\left(\mathrm{Mm}^{3}\right)$, dont une partie se perd par évaporation et une autre va grossir les oueds et les rivières et finit dans la mer Morte :

Tableau 1 : Pluviométrie annuelle (1986-1990)

\begin{tabular}{|c|c|c|}
\hline anne & quantite (Mm3) & \% changement \\
\hline $\begin{array}{l}1986 / 87 \\
1987 / 88 \\
1988 / 89 \\
1989 / 90\end{array}$ & $\begin{array}{r}6,700 \\
12,252 \\
10,205 \\
7,609\end{array}$ & $\begin{array}{r}- \\
55 \\
-20 \\
. \\
-34\end{array}$ \\
\hline moyenne & 9,191 & \\
\hline
\end{tabular}

La moyenne des précipitations annuelles varie entre $500 \mathrm{~mm}$ dans les zones semihumides, et moins de $100 \mathrm{~mm}$ dans les zones désertiques (cf. tableau $2 \mathrm{~d}$ 'après). Sur une surface totale de $92.600 \mathrm{~km} 2$, seuls $8,6 \%$ du pays reçoivent plus de $200 \mathrm{~mm} / \mathrm{an}$ de précipitations. $93 \%$ des zones cultivées sont irriguées par les eaux de pluie, les $7 \%$ restants par d'autres méthodes. Néanmoins, cette zone fournit environ $40 \%$ de la production agricole et $70 \%$ du produit agricole brut.

Tableau 2 : Répartition des sols selon la pluviométrie en 1990

\begin{tabular}{|l|c|c|c|}
\hline \multicolumn{1}{|c|}{ type } & pluviométrie (mm) & superficle (mdu) & pourcentage \\
\hline Aride désertique & 100 & 75.00 & 81.00 \\
Désertique & $100-200$ & 9.6 & 10.40 \\
Marginal & $200-300$ & 5.3 & 5.7 \\
Semi-aride & $300-500$ & 1.7 & 1.8 \\
Semi-humide & 500 & 1.0. & 1.1 \\
\hline Total & & 92,6 & 100 \\
& & & $\vdots$ \\
\hline
\end{tabular}

Source :

1- Ministère des Eaux et de l'irigation. Rapports annuels.

2 - Département des Terres et Cadastre, Amman - Jordanie.

100 Les ressources hydriques constituent le facteur-clé du développement socioéconomique jordanien. Un déficit ou une diminution de l'apport en eau grèverait lourdement l'effort de développement général. Par conséquent, développer le secteur hydrologique est crucial pour l'évolution des autres secteurs et figure en tête des priorités de la politique gouvernementale. Les décideurs et les planificateurs jordaniens 
sont soumis à d'énormes pressions pour pouvoir résoudre le problème que constitue la faiblesse des ressources hydriques. Situation aggravée par le retour en masse de quelque 400.000 travailleurs jordaniens et leurs familles des pays du Golfe après la crise $\mathrm{du}$ Golfe. Ce qui se traduit par une majoration de la demande annuelle en eau de 60 $\mathrm{Mm}^{3} / \mathrm{an}$.

En 1990, la consommation d'eau de la Jordanie s'est élevée à 800 millions de mètres cubes, provenant de deux sources principales et réparties comme suit :

- eaux de surface : $320 \mathrm{Mm}^{3}$, soit $40 \%$ du total des eaux disponibles, provenant surtout des deux fleuves, le Jourdain et le Yarmouk, de sources et d'oueds. Toutefois, le débit du Yarmouk s'est réduit à $386 \mathrm{Mm}^{3} / \mathrm{an}$, car la Syrie consomme 39\% de ses eaux et Israël 26\%, la Jordanie se contentant de $35 \%$.

- eaux souterraines : estimées à environ $480 \mathrm{Mm}^{3}$, soit $60 \%$ de l'ensemble des eaux disponibles. Elles proviennent des sources suivantes :

-a) renouvelables (alimentation pluviale de la nappe phréatique) ; $270 \mathrm{Mm}^{3}$, soit $56 \%$ de l'ensemble des eaux souterraines.

-b) fossiles (y compris les aquifères, considérées comme ressource hydrologique stratégique) : $210 \mathrm{Mm}^{3}$, soit $44 \%$ de l'ensemble des eaux souterraines. Les aquifères fournissent à la Jordanie environ $50 \mathrm{Mm}^{3}$ par an. A ce rythme, les eaux fossiles ne dureront que 100 ans.

La quantité totale des eaux souterraines renouvelables) exploitée en 1990 excède suivant :

103 Tableau 3 : Répartition des eaux souterraines renouvelables et non-renouvelables par secteur en 1990

\begin{tabular}{|c|c|c|}
\hline répartition & quantlté (Mm3) & \% changement \\
\hline $\begin{array}{l}\text { Eaux potables } \\
\text { Eaux industrielles } \\
\text { Eaux agricoles }\end{array}$ & $\begin{array}{r}165 \\
35 \\
188\end{array}$ & $\begin{array}{r}42.5 \\
9.0 \\
48.5\end{array}$ \\
\hline Total & 388 & 100 \\
\hline
\end{tabular}

\section{Source : Dossiers du Ministère des Eaux el de limigation.}

Pour augmenter les réserves hydriques l'État a construit de nombreux barrages, dont la capacité totale varie de 115 à $120 \mathrm{Mm}^{3}$, mais leur débit n'a été que de $65 \mathrm{Mm}^{3}$ en 1990 à cause de la faible d'où une réduction de 5 à $10 \%$ des eaux d'irrigation dans la vallée du Jourdain. Le futur barrage de l'Unité sur le Yarmouk, permettrait d'espérer un débit de plus de $250 \mathrm{Mm}^{3}$ pour irriguer la vallée. Mais il ne semble pas près de voir Ie jour, la plupart des bailleurs de fonds répugnant à se lancer dans un projet qui nécessite l'accord des Israéliens.

\section{Utilisation des eaux}

En 1990, environ 99\% de la population jordanienne avait accès à l'eau potable. De 1990 à 2005, la consommation totale des eaux en Jordanie passera de $730 \mathrm{Mm}^{3}$ à $1.120 \mathrm{Mm}^{3}$, soit une majoration de plus de $65 \%$. 
Tableau 4 : Répartition de la consommation d'eau par secteur en 1990 et 2005

\begin{tabular}{|l|c|r|c|c|}
\hline Secteur & $1990(\mathrm{Mm} 3)$ & $\%$ & $2005(\mathrm{Mm} 3)$ & $\%$ \\
\hline Domestique & 175 & 24.0 & 300 & 26.8 \\
Industriel & 35 & 4.8 & 70 & 6.3 \\
Agricole & 520 & 71.2 & 750 & 67.0 \\
& 730 & 100 & & 100 \\
Total & & & \\
\hline
\end{tabular}

Selon les estimations, la consommation jordanienne d'eau per capita est d'environ 230 $\mathrm{m}^{3}$ par an, avec une augmentation potentielle de $250 \mathrm{~m}^{3}$ si les ressources en eau sont bien mises en valeur. Chiffre qui demeure bien en-deçà du seuil mondial de pauvreté en eau : $1.000 \mathrm{~m}^{3}$ per capita/an.

\section{Consommation agricole}

En 1990, le secteur agricole a consommé $520 \mathrm{Mm}^{3}$ d'eau, soit $71 \%$ de la consommation totale de la Jordanie. Dans ce pays, les terres irriguées dépassent 700.000 dunums (175.000 acres), dont $46 \%$ se trouvent dans la Vallée du Jourdain, donc irriguées par le Yarmouk et les oueds du Jourdain. Toutefois, ces 700.000 dunums pourraient recevoir tous les ans $340 \mathrm{Mm}^{3}$ par une irrigation au goutte-à-goutte, ce qui épargnerait au pays environ $180 \mathrm{Mm}^{3}$. Quantité qui dépasserait la consommation domestique de 1990.

La fluctuation des précipitations est déterminante pour la planification des fermiers. Un déficit de pluviosité réduira l'irrigation et, par suite, les parcelles cultivées lorsque les fermiers ne pourront plus assurer l'irrigation en été. Aussi des restrictions ont-elles été imposées aux fermiers par l'État en ce qui concerne tes types de cultures. En Jordanie, les experts agronomes devraient tabler sur l'hypothèse de deux années de sécheresse pour chaque cycle de quatre ans, seul moyen d'adapter le développement aux réalités d'un climat sec.

Les eaux d'irrigation proviennent des sources indiquées sur le tableau suivant : 
Tableau 5 : Sources des eaux d'irrigation en 1990

\begin{tabular}{|l|c|c|}
\hline \multicolumn{1}{|c|}{ source } & quantité (Mm3) & pourcentage \\
\hline Canal du Roi Abdallah & 130 & 25.00 \\
Oueds nord Ghor & 80 & 15.00 \\
Oueds sud Ghor & 40 & 7.00 \\
Région Disi & 50 & 10.00 \\
Usines traitement eaux & 30 & 5.80 \\
Puits secteur privé & 190 & 36.50 \\
& & 100 \\
Total. & 520 & \\
\hline
\end{tabular}

Source : Dossiers du Ministère des Eaux et de Pirrigation.

\section{Consommation domestique}

110 Elle atteint en Jordanie $175 \mathrm{Mm}^{3}$, soit 4\% de la consommation totale des eaux en 1990. Avec une croissance démographique moyenne de $3.6 \%$, la consommation d'eau atteindra $300 \mathrm{Mm}^{3}$ en l'an 2000 lorsque la population passera à 5,46 millions. La consommation domestique moyenne per capita en eaux à usage ménager est d'environ $53 \mathrm{~m}^{3}$ /an. La Jordanie devra doubler son volume d'eau disponible au cours des vingt prochaines années pour répondre à la croissance démographique.

Tableau 6 : Consommation domestique

\begin{tabular}{|c|c|c|c|c|c|}
\hline amee & $\begin{array}{c}\text { quantite } \\
\text { (Mm3) }\end{array}$ & $\begin{array}{c}\text { population } \\
\% \\
\text { changem }\end{array}$ & $\begin{array}{c}\text { poputation } \\
(000)\end{array}$ & $\begin{array}{c}\% \\
\text { changem }\end{array}$ & $\begin{array}{l}\text { corsomm } \\
\text { per caplta } \\
\text { (m3) }\end{array}$ \\
\hline 1986 & 135 & • & $2,796.1$ & - & 48.3 \\
\hline 1987 & 150 & 11.00 & $2,896.6$ & 3.59 & 51.8 \\
\hline 1988 & 165 & 11.00 & $3,000.1$ & 3.57 & 55.0 \\
\hline 1989 & 172 & 4.2 & $3,111.0$ & 3.69 & 55.3 \\
\hline 1990 & 175 & 1.7 & $3,226.0$ & 3.70 & 54.2 \\
\hline 2005 & 300 & 17.1 & $5,460.0$ & 59.00 & 55.0 \\
\hline
\end{tabular}


111 Face à une demande croissante en été, le ministère des Eaux et de l'Irrigation a entamé un programme de rationnement de l'eau, bien que les Jordaniens soient de petits consommateurs par rapport aux populations des autres pays de la région.

\section{Consommation industrielle}

En 1990, le secteur industriel a consommé $35 \mathrm{Mm}^{3}$ d'eau, coefficient de consommation le plus faible par rapport aux autres secteurs mais également indice de l'activité économique du pays. Au sein de ce même secteur, les plus gros consommateurs d'eau sont les industries minières, énergétiques, les usines de papier. Récemment, la question des eaux industrielles polluées a fait couler beaucoup d'encre en Jordanie. Le gouvernement a pris des mesures conservatoires à l'égard de plus de quarante sociétés accusées d'avoir pollué des cours d'eau, surtout le barrage du Roi Talal. Elles devront mettre en place des unités de traitement des eaux ou fermer leurs portes.

En Jordanie, le prix de l'eau varie en fonction de la situation géographique et des quantités consommées. Trois paramètres différents déterminent la fixation des prix :

- prix de base minimum : montant destiné à couvrir une partie des prix de revient. Le prix de base minimum est calculé selon un volume d'eau minimum $\left(\mathrm{en}^{3}\right.$ ), consommé ou non. Le volume minimum pour la grille actuelle des prix est de $60 \mathrm{~m}^{3} / \mathrm{an}$;

- éventail des eaux consommées : un ensemble de critères a été mis en place pour identifier les eaux consommées. Un cloisonnement entre les différents types de consommation a été établi en fonction du revenu, de la situation géographique et des fins de l'utilisation ;

- proportion d'eau consommée : le prix de l'eau n'est pas linéaire. Lors de l'élaboration des grandes lignes de la politique des prix, la structure sociale, les fins de l'utilisation et les moyens de réduire la consommation sont pris en considération. En conséquence, le prix de l'eau est beaucoup plus élevé lorsque la consommation dépasse un certain quota.

114 Le ministère des Eaux et de l'Irrigation alimente les abonnés en eau pour des usages divers, et le prix de l'eau est fonction de cet usage :

- usage domestique : le prix démarre à 0,065 dinar jordanien/mètre cube $\left(\mathrm{DJ} / \mathrm{m}^{3}\right)$;

- usage industriel : les prix vont de 0,30 à $0,85\left(\mathrm{DJ} / \mathrm{m}^{3}\right)$;

- usage agricole : 0,006 DJ/m³ (prix subventionnés).

\section{En conclusion}

La Jordanie, pays proche-oriental le plus sévèrement atteint par la pénurie d'eau, devra affronter le problème aux plans local et régional.

Sur le plan local

- Une coopération s'impose entre la Jordanie et d'autres pays de la région en vue du démarrage du projet " pipe-line de la paix », à partir de la Turquie. Ce projet, qui ne représente que $15 \%$ des dépenses engagées dans la guerre du Golfe, renforcerait la coopération entre les différents bénéficiaires du pipe-line.

- La Jordanie n'étant pas un pays exportateur de pétrole, des usines conjointes de dessalement devraient être mises en place, permettant ainsi le partage des coûts et de la technologie. Une petite usine nucléaire pourrait entreprendre cette opération.

- La Jordanie pourrait partager avec des pays voisins la technologie relative à la prospection et à l'utilisation de l'eau à l'issue d'un règlement pacifique des conflits dans la région. 
- La Jordanie pourrait également importer de l'eau, par exemple d'Irak (Euphrate) et d'Égypte (Nil).

117 Sur le plan régional

- Gestion et optimisation des ressources hydrologiques renouvelables en vue de répondre aux besoins croissants.

- Utilisation judicieuse et préservation des eaux comme suit :

118 a) substitution, à l'irrigation en surface, d'une irrigation souterraine (tuyaux placés dans le sol) qui permettrait d'économiser 35\% de la consommation hydrique ;

119 b) entretien et amélioration du réseau national de tuyaux, économisant ainsi $20 \%$ de la consommation ;

c) recyclage des eaux usées en vue de leur réutilisation à des fins agricoles et industrielles ;

121 d) majoration du prix des eaux destinées aux secteurs agricole et industriel pour les aligner sur les coûts de fonctionnement/entretien du ministère des Eaux et de l'Irrigation ;

e) variation du prix des eaux d'irrigation en fonction des récoltes ;

f) Le secteur agricole consommant $71 \%$ du total des eaux utilisées, les agriculteurs devront prendre part au processus de prise de décision concernant le fonctionnement et l'entretien du réseau d'irrigation;

$124 \mathrm{~g})$ les firmes industrielles doivent assumer les frais d'épuration des ressources hydrologiques polluées ;

h) la JVA (Jordan Valley Authorithy) devrait pomper les eaux du Canal du Roi Abdallah vers les barrages durant la saison d'hiver.

- Encourager la construction de barrages-réservoirs dans le désert, dans la mesure du possible.

- Publier les déficits en eau afin de mieux faire partager la responsabilité de la préservation des eaux et de sensibiliser le public.

Traduit de l'anglais par Nefissa El Bakly.

\section{INDEX}

Mots-clés : droit islamique, eau, Israël, Jordanie, Turquie 